\title{
Spatially Explicit Assessment of Ecosystem Resilience: An Approach to Adapt to Climate Changes
}

\author{
Haiming Yan, ${ }^{1}$ Jinyan Zhan, ${ }^{1}$ Bing Liu, ${ }^{2}$ Wei Huang, ${ }^{3}$ and Zhihui $\mathrm{Li}^{4,5,6}$ \\ ${ }^{1}$ State Key Laboratory of Water Environment Simulation, School of Environment, Beijing Normal University, Beijing 100875, China \\ ${ }^{2}$ College of Geomatics, Shandong University of Science and Technology, No. 579 Qianwangang Road, \\ Economic \& Technical Development Zone, Qingdao, Shandong 266590, China \\ ${ }^{3}$ Department of Agricultural Economics and Rural Development, Georg-August-Universität Göttingen, Raum MZG 2016, \\ Platz der Göttinger Sieben 5, 37073 Göttingen, Germany \\ ${ }^{4}$ Institute of Geographic and Natural Resources Research, Chinese Academy of Sciences, Beijing 100101, China \\ ${ }^{5}$ University of Chinese Academy of Sciences, Beijing 100049, China \\ ${ }^{6}$ Center for Chinese Agricultural Policy, Chinese Academy of Sciences, Beijing 100101, China
}

Correspondence should be addressed to Jinyan Zhan; zhanjy@bnu.edu.cn

Received 2 November 2013; Revised 4 January 2014; Accepted 11 January 2014; Published 19 February 2014

Academic Editor: Dong Jiang

Copyright (C) 2014 Haiming Yan et al. This is an open access article distributed under the Creative Commons Attribution License, which permits unrestricted use, distribution, and reproduction in any medium, provided the original work is properly cited.

\begin{abstract}
The ecosystem resilience plays a key role in maintaining a steady flow of ecosystem services and enables quick and flexible responses to climate changes, and maintaining or restoring the ecosystem resilience of forests is a necessary societal adaptation to climate change; however, there is a great lack of spatially explicit ecosystem resilience assessments. Drawing on principles of the ecosystem resilience highlighted in the literature, we built on the theory of dissipative structures to develop a conceptual model of the ecosystem resilience of forests. A hierarchical indicator system was designed with the influencing factors of the forest ecosystem resilience, including the stand conditions and the ecological memory, which were further disaggregated into specific indicators. Furthermore, indicator weights were determined with the analytic hierarchy process (AHP) and the coefficient of variation method. Based on the remote sensing data and forest inventory data and so forth, the resilience index of forests was calculated. The result suggests that there is significant spatial heterogeneity of the ecosystem resilience of forests, indicating it is feasible to generate largescale ecosystem resilience maps with this assessment model, and the results can provide a scientific basis for the conservation of forests, which is of great significance to the climate change mitigation.
\end{abstract}

\section{Introduction}

There is ample evidence of the ecological impacts of climate change, which may pose considerable challenges to the terrestrial ecosystems and change the provided ecosystem services in the future $[1,2]$, and the forest ecosystem resilience plays an important role in maintaining the desirable ecosystem states that allow these ecosystem services to be delivered, and enables quick and flexible responses to climate changes [3]. For example, forests are major reservoirs of terrestrial biodiversity and contain about $50 \%$ of the global terrestrial biomass carbon stocks, emissions from deforestation and degradation remain a significant source of annual greenhouse gas emissions into the atmosphere, and therefore the conservation, appropriate management, and restoration of forests will make a significant contribution to climate change mitigation [4]. Resilience is the capacity of an ecosystem to withstand external pressures and return to its predisturbance state over time, the loss of ecosystem resilience indicates that ecosystems are prone to the shifts to undesirable states in which the ecosystem services needed by humans can no longer be delivered, and maintaining or restoring the forest ecosystem resilience is often cited as a necessary societal adaptation to climate change $[5,6]$. However, forest ecosystem resilience has continually declined at the regional scale and even global scale due to the climate change and human disturbance [7]. The quantitative assessment of ecosystem resilience can provide a scientific basis for the forest resource 
management and conservation and therefore is of great significance to the maintenance of critical ecosystem services [8].

The concept of resilience has been widely used, and there have been some ecological theories that attempt to explain the mechanism of resilience through a variety of models $[9,10]$, for example, "species richness-diversity" [11], "functional redundancy" [12], "keystone species hypothesis" [13], "resilience-productivity hypothesis" [14]. However, these theories are generally based on the concept of species populations as the basic functional unit and therefore fail to capture the importance of the interactions amongst individual organisms in the ecosystem [10]. Among the current theories, the theory of dissipative structures seems particularly suitable for investigating the dynamics of structural change and resilience of ecosystems $[15,16]$. It shows that the open and self-organizing systems maintain their structural order by keeping their internal state far from thermodynamic equilibrium through active exchanges with their environment [16]. Those dissipative structures are in principle stable as long as the exchanges with the environment are maintained and the continuous perturbations are absorbed within the framework of the given dynamic regime $[15,16]$. The theory of dissipative structures provides a scientific theoretical framework for explaining the mechanism of the ecosystem resilience; however, there have been very few researches on the quantitative measurement of ecosystem resilience on the basis of this theory; more in-depth research should be carried out on how to more scientifically and accurately assess the ecosystem resilience with reasonable indicators of the ecosystem resilience.

Resilience can be measured in terms of change in a system level property and function following perturbation, and the perturbation can be simulated [10]. In previous research, the ecosystem resilience was generally measured by the rate of return of the ecosystem state after disturbance or the maximum disturbance that the ecosystem can absorb before shifting to another state [17]. Currently, researchers generally select one key indicator associated with the ecosystem, for example, the key species and vigor of the ecosystem, and they then simulate the time for the key indicator to return from the stressed state to the normal state ( $\mathrm{Tr}$ ) and the maximum stress that the ecosystem can withstand (MS) with models such as the CENTRURY model and the GAP model [18]. Ecosystem resilience can be represented by the values of MS, $1 / \operatorname{Tr}$, or MS/Tr [19]. This approach assumes that the dynamics of the ecosystem can be understood by analyzing a few key variables, which is termed the "rule of hand" [5]. However, the concept of "rule of hand" is limited and relatively unrepresentative because it is impossible to represent the complete recovery of ecosystem function by the recovery of only a few key variables [20]. In addition, although it is in principle possible to measure ecosystem resilience by fitting a dynamic model to time series, this approach imposes extraordinary data requirements. It is usually difficult to obtain the data that can meet such requirements in practice [17].

It is more plausible to measure resilience in terms of the factors influencing resilience. The literature reported a number of factors that influence the ecosystem resilience, for example, the diversity within functional groups and variability of habitats [21]. However, these factors have not previously been considered comprehensively by researchers. The operational indicators of resilience have received little attention in the literature, and there is no consensus-based view of how to measure resilience or even of the exact nature of resilience [22]. Rosset and Oertli assessed the resilience of species to warming with five ecological and biogeographical metrics and explained their theoretical basis [23]; however, this approach may be relatively biased since these researchers simply used equal weights for each metric.

This study aims to develop a conceptual framework for the spatially explicit assessment of the forest ecosystem resilience based on the theory of dissipative structures, and the rest of this paper is organized as follows. The second part presents a brief overview of the study area and explains how the indicator system was constructed, how the indicator weights were determined, and how the resilience index was calculated. Besides, this part also shows the data used in this study and how they were processed. The third part presents the results and discusses the underlying reasons for the spatial heterogeneity of the forest ecosystem resilience, and the final part concludes.

\section{Data and Methodology}

2.1. Study Area. Yongxin County is representative of the subtropical mixed conifer and broadleaved forest area in the Poyang Lake watershed, with a forest area of 143,980 ha and a forest coverage rate of $65.6 \%$. It is located between $26^{\circ} 47^{\prime}$ $27^{\circ} 14^{\prime} \mathrm{N}$ and $113^{\circ} 50^{\prime}-114^{\circ} 29^{\prime} \mathrm{E}$, in the upper and middle reaches of the Heshui River which is the largest secondary tributary of Poyang Lake (Figure 1). The northern and southern parts of Yongxin County primarily include mountains and hills, where there are a lot of forests, whereas the central part of the county includes hills and plains, most of which are covered by cultivated land. It is the subtropical monsoon climate in this region, with an annual average temperature of $18.2^{\circ} \mathrm{C}$ and an annual average precipitation of $1,530.7 \mathrm{~mm}$, and the zonal vegetation is the evergreen broadleaved forest, but the existing forests primarily consist of Pinus massoniana and Cunninghamia lanceolata (C. lanceolata), and the current state of the forests is the result of both the long-term human disturbance and restoration and the natural recovery under the influence of the regional natural background [24].

2.2. Data and Processing. The data used primarily include the forest inventory data, remotely sensed data, and statistical data. The forest inventory data in 2009 were obtained from the Yongxin Forestry Bureau, including the forest resource distribution map, data for 707 sample plots, and data for 41603 sample trees. We obtained the forest distribution map by integrating the forest form map with the Landsat Thematic (TM) image covering the study area. Besides, we interpolated the sample plot data and sample tree data into $30 \mathrm{~m} \times$ $30 \mathrm{~m}$ grid data with the Kriging method to obtain the data of specific indicators of the internal memory, such as the average stand age, average $\mathrm{DBH}$ growth rate, and mature 




FIGURE 1: Location of Yongxin County and the distribution map of forests.

dominant tree density [25]. The external memory was then calculated on the basis of the internal memory. Besides, the factor influencing the availability of the external memory was indicated with the proximity index, which was calculated on the basis of the forest distribution map. In addition, the data of indicators of the stand conditions were prepared with the remote sensing data, observation data, and statistical data. For example, the climatic data were from the observation stations in Jiangxi Province and Hunan Province maintained, and the original data were interpolated into $30 \mathrm{~m} \times 30 \mathrm{~m}$ grid data using the gradient plus inverse distance squares method and then extracted the part of Yongxin County. The soil data were extracted from the forest inventory data of the second nationwide general soil survey and were interpolated into $30 \mathrm{~m} \times 30 \mathrm{~m}$ grid data with the Kriging method [26]. The terrain data were obtained with the $30 \mathrm{~m} \times 30 \mathrm{~m}$ digital elevation model (DEM) data. The distance to the nearest river was obtained from the 1:250,000 topographic maps of Jiangxi Province. The population data were obtained from China Population Statistics Yearbook 2010 and were spatially disaggregated into $30 \mathrm{~m} \times 30 \mathrm{~m}$ grid data with the spatial disaggregating method [27].

2.3. Model Development. The forest ecosystem resilience is quantitatively measured with its influencing factors on the basis of the theory of dissipative structures in this study. First, some definitions related to the ecosystem resilience were clarified, which lay the foundation for the ecosystem resilience assessment. Then based on the theory of dissipative structures, a hierarchical indicator system was constructed according to the factors influencing the forest ecosystem resilience, most of which have some impacts on the energy and material flows between the forest ecosystem and the environment. Thereafter, the indicator weights were determined with the combined weighting method, including the analytic hierarchy process (AHP) and coefficient of variance (CV) method [28]. Finally, the resilience index of forests was calculated as the weighted sum of these indicators at the grid scale.

2.3.1. Definition of Ecosystem Resilience, State, and Scale. It is necessary to make some definitions related to the ecosystem resilience so as to make the results comparable. First and foremost, it is necessary to clarify the definition of ecosystem resilience since the literature offers various definitions of ecosystem resilience and includes a controversy about the existence of different ecosystem states [16]. The current viewpoints can be summarized into ecological resilience and engineering resilience, and forests are engineering resilient in the sense that they may in time return to their predisturbance state and maintain approximately the original species composition [3]. Therefore the definition of the engineering resilience has been adopted in this study, referring to the capacity of an ecosystem to absorb disturbance and return to its predisturbance state following a perturbation [29].

Secondly, it is crucial to specify the ecosystem state of interest since the forest ecosystems have multiple states under which different ecosystem services may be delivered [17]. This study has only focused on the resilience under the current state, which is defined with the dominant tree species [3], and it is assumed that there will be no state transformation during the study period. In addition, it is necessary to specify the scale in the resilience assessment since the different scales may lead to different assessment results [30, 31], and when viewed over an appropriate time span, a resilient forest ecosystem is able to maintain its "identity" in terms of taxonomic composition, structure, ecological functions and process rates [3]. As for the spatial scale, this study proposes to measure the absolute and relative conditions (e.g., space, environmental characteristics, and resource availability) at the patch scale and to analyze questions of resilience at the multipatch scale [31], and the $30 \mathrm{~m}$ resolution patch has been used to analyze the resilience in this study. As for the time scale, the forest ecosystem resilience is analyzed at the annual scale, and it is assumed that no ecosystem state transformations would occur during the study period since the ecosystem generally fluctuates near equilibrium and remains essentially stable during a given period.

2.3.2. Indicator System. A hierarchical model was developed with factors influencing the forest ecosystem resilience according to the theory of dissipative structures. The theory of dissipative structures shows that the forest ecosystem as a system of dissipative structures will soon collapse on condition that there is no input of energy and material [15], and the ecosystem resilience depends on both the favorable stand conditions and the biological and ecological resources in the ecosystem, all of which influence the input of energy and material into the forest ecosystem. Therefore the resilience index that evaluates the forest ecosystem resilience was disaggregated into the stand conditions and the ecological memory, which were finally disaggregated into simple indices that are measurable and can be observed in the field. 
The indicators of the stand conditions include the terrain, soil, climate, water conditions, and human disturbance, which interact with each other and jointly affect the resilience of the forest ecosystem [32]. The terrain has obvious impacts on the factors required for plant growth, for example, the water and soil nutrients. For example, there is richer soil fertility and seed bank in the lower slope position than the middle and upper slope positions, and the seed germination rate at the lower slope position is higher [33], and the terrain indicators in this study include the aspect, slope, and slope position. Besides, the resilience at the local level depends on the ability of the landscape to maintain infiltration, water storage capacity and nutrient cycles, all of which are threatened by soil loss and structural change [34]. In this study, we selected the soil depth, humus horizon depth, and loam quantity for use as soil structure indices and used the humus quantity, soil nitrogen $(\mathrm{N})$ quantity, soil phosphorus (P) quantity, and soil potassium $(\mathrm{K})$ quantity as indices of soil fertility. In addition, the climate primarily influences photosynthesis, respiration, and other ecosystem processes through medium-term and long-term temperature, radiation and wetness and consequently exerts great impacts on the plant growth [4], and water conditions also have significant influence on plant growth through influencing the availability of water [35]. The climate indices used in this study included the annual accumulated temperature above $10^{\circ} \mathrm{C}$, the annual precipitation, and the annual hours of sunshine, and the distance to the nearest river was used as the indicator of the water conditions. What is more, there is still controversy over the identification of humans as a component of natural ecosystems, but human beings have altered the resilience of ecosystems [36]; therefore the population density that is closely related with human activities has been used as the indicator of human disturbance in this study.

The ecological memory include the internal memory within the foci forest patch and external memory in the neighbor patches of the foci forest patch [37, 38], which were further represented with more specific indicators. The internal memory includes the species that survive within the disturbed area (i.e., the biological legacies) and the remaining dead organic structures that serve as foci for regeneration and allow species to colonize (i.e., the structural legacies) [38]. The biological legacies include the seed, vegetation materials, and animal communities, while the structural legacies provide critical protective cover, habitat, and food and nutrient sources for a variety of organisms and influence geomorphic processes such as erosion and the deposition of sediments [38]. First, the seed bank is the material basis for the natural regeneration of forests [37]. Since it is difficult to measure the soil seed bank in a large area, we used the factors influencing the soil seed bank as the specific indicators, including the density of the mature dominant trees and the stand canopy [39]. Besides, the ecosystem resilience resides in both the diversity of the drivers and the number of passengers who are potential drivers, which are of different significance to the ecosystem [40], and the selected indictors of the diversity of the drivers include the species number of the dominant trees and the subdominant trees and the grass and shrub canopy. In addition, ecophysiological characteristics of the vegetation also contribute to ecosystem resilience [3], the indicators of which include the average stand age and the average growth rate of the diameter at breast height $(\mathrm{DBH})$ in this study. What is more, structural legacies provide critical protective cover that allows species to colonize [21], and the litter depth was used as the indicator of structural legacies in this study. More importantly, the recovery of an ecosystem from disturbance requires an area that is sufficiently large and abundant internal memory to guarantee a rapid reorganization, and a larger and less fragmented forest ecosystem is more resilient [3]. In this study, the patch area and perimeter-to-area ratio were used to represent the patch size and patch shape, respectively.

Ecosystem reorganization requires both the internal memory within the disturbed patch and the external memory within neighboring patches, which provides seed flows among forest patches and influence the species composition and facilitate the resilience of disturbed patches [28]. Since it is difficult to measure long-distance seed dispersal, we measured the external memory by the density of mature dominant trees within neighboring patches of corresponding patch types whose edges are within a specified distance of the focal patch (1000 $\mathrm{m}$ in this study). In addition, many plant species are dispersal limited and are influenced by various factors such as the distance to the seed sources and the availability of dispersal agents [41], and therefore we have used a proximity index that combines the area of the neighboring patch and the distance to the focal patch as the indicator of the factors that influence the availability of external memory (Table 1).

2.3.3. Calculation of the Resilience Index. There are many methods to synthetize the basic indicators into one index, such as the fuzzy comprehensive evaluation method, Delphi method, and comprehensive index method [42]. This study used the classic comprehensive index method to calculate the resilience index of the forest ecosystem. First, the assessment indicators of the forest ecosystem resilience were assigned with different weights; then the weighted sum of the assessment indices was calculated with a spatial overlay of these indices. The resilience of the forest ecosystem is calculated with the following formula:

$$
\text { resilience }_{j}=\sum_{i=1}^{n} w_{i} x_{i}, \quad(i=1,2,3, \ldots, n),
$$

where resilience ${ }_{j}$ is the resilience index of the $j$ th assessment unit, $w_{i}$ is the weight of the $i$ th assessment index, $x_{i}$ is the value of the $i$ th assessment index, which is normalized with the extreme value method, and $n$ is the number of the assessment indices.

Besides, there are many methods to determine the index weights, which can be generally classified into the subjective weighing method and objective weighing method, both of which have some disadvantages $[43,44]$. The combinatorial weighing method was used to determine the indicator weights to reduce the possible errors in this study. The indicator weights were first determined with AHP and the coefficient of variation method and then combined as follows 
TABLE 1: The assessment system and weights of specific indicators. $W_{\mathrm{AHP}}$ and $W_{\mathrm{CV}}$ refer to the weights determined with the analytic hierarchy process (AHP) and coefficient of variation method (CV), respectively. The weights were calculated as the average of $W_{\mathrm{AHP}}$ and $W_{\mathrm{CV}}$.

\begin{tabular}{|c|c|c|c|c|}
\hline Medium-level indicators & Bottom indicators & $W_{\mathrm{AHP}}$ & $W_{\mathrm{CV}}$ & Weights \\
\hline \multicolumn{5}{|l|}{ Stand conditions } \\
\hline \multirow{3}{*}{ Terrain } & Slope position & 0.0123 & 0.0386 & 0.0254 \\
\hline & Slope & 0.0088 & 0.0209 & 0.0148 \\
\hline & Aspect & 0.0257 & 0.0417 & 0.0337 \\
\hline \multicolumn{5}{|l|}{ Climate } \\
\hline \multirow{2}{*}{ Temperature } & Annual average temperature & 0.0138 & 0.0503 & 0.0320 \\
\hline & Cumulative temperature above $10^{\circ} \mathrm{C}$ & 0.0292 & 0.0543 & 0.0417 \\
\hline Precipitation & Annual precipitation & 0.0356 & 0.0289 & 0.0322 \\
\hline Solar radiation & Annual sunshine hour & 0.0102 & 0.0467 & 0.0284 \\
\hline Water condition & Distance to the nearest river & 0.0194 & 0.0075 & 0.0134 \\
\hline \multicolumn{5}{|l|}{ Soil } \\
\hline \multirow{3}{*}{ Soil structure } & Soil depth & 0.0063 & 0.0177 & 0.0120 \\
\hline & Humus depth & 0.024 & 0.0168 & 0.0204 \\
\hline & Loam quantity & 0.0035 & 0.00001 & 0.0017 \\
\hline \multirow{4}{*}{ Soil fertility } & Soil organic quantity & 0.0045 & 0.0073 & 0.00591 \\
\hline & Soil nitrogen quantity & 0.0065 & 0.0016 & 0.00406 \\
\hline & Soil phosphorus quantity & 0.0036 & 0.00001 & 0.0018 \\
\hline & Soil kalium quantity & 0.0044 & 0.0016 & 0.00301 \\
\hline Human disturbance & Population density & 0.0237 & 0.0273 & 0.0255 \\
\hline \multicolumn{5}{|l|}{ Ecological Memory } \\
\hline \multicolumn{5}{|l|}{ Internal memory } \\
\hline \multirow{6}{*}{ Biological legacies } & Species number of dominant and subdominant trees & 0.0459 & 0.0824 & 0.0641 \\
\hline & Grass-shrub canopy & 0.0145 & 0.0308 & 0.0226 \\
\hline & Average stand age & 0.0293 & 0.0106 & 0.0199 \\
\hline & Average $\mathrm{DBH}$ growth rate & 0.0206 & 0.0342 & 0.0274 \\
\hline & Mature dominant tree density & 0.0568 & 0.0487 & 0.0527 \\
\hline & Vegetation canopy & 0.0171 & 0.0257 & 0.0214 \\
\hline Structural legacies & Litter depth & 0.0828 & 0.0384 & 0.0606 \\
\hline \multirow{2}{*}{ Patch area and shape } & Patch area & 0.2487 & 0.1001 & 0.1744 \\
\hline & Perimeter area ratio & 0.0749 & 0.0058 & 0.0403 \\
\hline \multirow{2}{*}{$\begin{array}{l}\text { External memory and factors } \\
\text { influencing its availability }\end{array}$} & $\begin{array}{l}\text { Density of mature dominant tree within neighbor } \\
\text { patches of corresponding types }\end{array}$ & 0.0552 & 0.0665 & 0.0608 \\
\hline & Proximity index & 0.1227 & 0.1949 & 0.1588 \\
\hline
\end{tabular}

(see (2)) in order to take full advantage of expert knowledge and give full consideration to specific conditions of the study area:

$$
w_{i}=\frac{w_{j}^{(1)} \times w_{j}^{(2)}}{\sum_{j=1}^{m}\left[w_{j}^{(1)} \times w_{j}^{(2)}\right]},
$$

where $w_{j}^{(1)}$ is the weight vector obtained with the coefficient of variation method, $w_{j}^{(2)}$ is the weight vector obtained with AHP, and $j$ is the number of assessment indices. The final index weights are listed in Table 1.

\section{Results and Discussion}

3.1. Result of the Assessment of Resilience. The indicator system was finally established and indicator weights were determined (Table 1), with which the forest ecosystem resilience in the study area was calculated and was further divided into five levels (Figure 2), with the thresholds of the resilience index determined with the natural breaks method (Table 2).

The forests with the highest resilience were mainly located in the middle of the southwestern mountain area. The result indicated that the forest ecosystem resilience in Yongxin County ranged from 0.1803 to 0.6919 , with an average value of 0.3821 . Only $40.4 \%$ of the forests were above the average resilience level, and the forest ecosystem resilience in the 

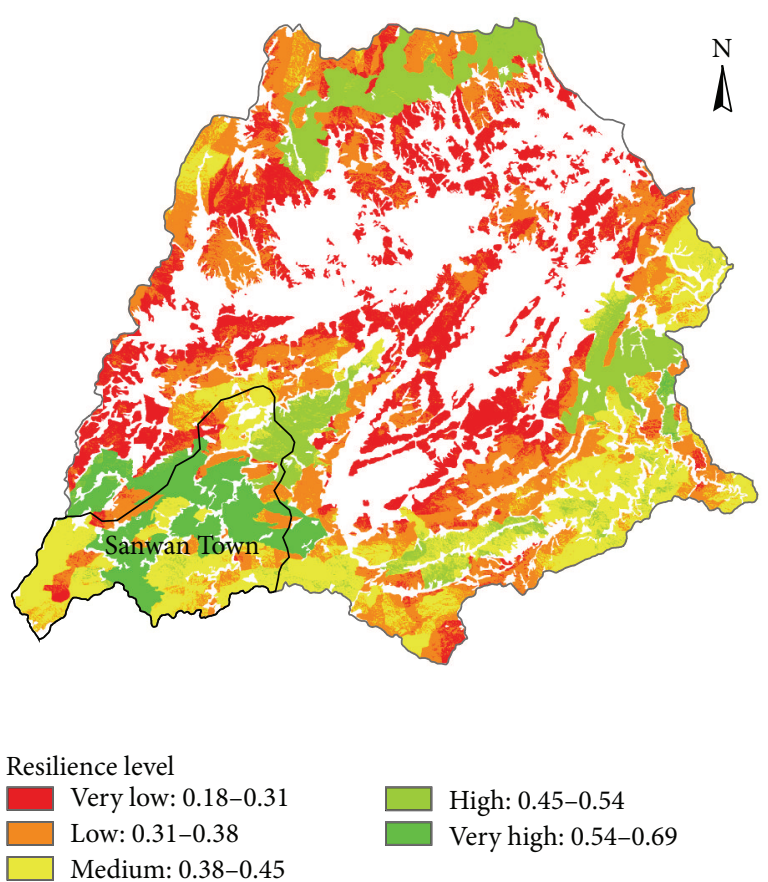

FIgURE 2: Spatial pattern of the forest ecosystem resilience level in Yongxin County and the location of Sanwan Town where Sanwan National Forest Park is located.

study area was not very high on the whole. Besides, the forest ecosystem resilience is generally above the average level occurred in the southwestern mountain area, the southeastern mountain area, and the northern mountain area, while the resilience was generally below the average level in the middle plain and hill area. The map of the forest ecosystem resilience level clearly showed the spatial heterogeneity of the pattern of resilience (Figure 2). The forests reaching a high or very high resilience level generally occurred around the center of the southwestern mountain area and the eastern part of the southeastern mountain area, and they extended from east to west in the northern mountain area. The forests with a medium level of resilience generally occurred in the area of the southern boundary, whereas the forests with a low or very low level of resilience were generally located in the central plain and hill area. The forests in the southwestern mountain area generally reached or exceeded a medium level of resilience, with a large area reaching the very high resilience level. The forests only reached a medium or low level of resilience in the southeastern mountain area except in the eastern part and the area near the administrative boundary, where the forests reached a high level of resilience. In the northern mountain area, only the forests extending from east to west, whose type species was C. lanceolata, reached a medium or high level of resilience. The remainder of the forests in the northern mountain area only reached a low or very low level of resilience. In contrast, the forests in the central area were almost entirely at a low or very low level of resilience.

The total forest area at each resilience level was furthermore summarized (Table 2). In total, $57.5 \%$ of the forests
TABLE 2: Threshold of forest resilience level and total area at each resilience level.

\begin{tabular}{lccc}
\hline Resilience level & $\begin{array}{c}\text { Threshold of } \\
\text { resilience levels }\end{array}$ & Total area (ha) & $\begin{array}{c}\text { Area percent } \\
(\%)\end{array}$ \\
\hline Very low & $0.18-0.31$ & 35835.93 & 25.60 \\
Low & $0.31-0.38$ & 44688.15 & 31.90 \\
Medium & $0.38-0.45$ & 30632.22 & 21.90 \\
High & $0.45-0.54$ & 18448.29 & 13.20 \\
Very high & $0.54-0.69$ & 10386.63 & 7.40 \\
\hline
\end{tabular}

were at a low or very low level of resilience; $21.9 \%$ reached a medium level of resilience. Only $20.6 \%$ reached or exceeded a high level of resilience, indicating the resilience of the forest was not good. Besides, only five forest types reached the very high resilience level, among which the $C$. lanceolata forest accounted for the largest area proportion of forests with very high resilience, with the total area of 10381.2 ha reaching the very high resilience level. In addition, many forest types reached the high resilience level, but only the area of C. lanceolata, other sclerophyllous forest, and Theaceae was relatively large, reaching 16,457.6 ha, 1,951.02 ha, and $12.42 \mathrm{ha}$, respectively. In summary, most forest types only reached a medium level of resilience, only the forest types with a large total area, such as C. lanceolata, tended to have large areas exceeding the medium resilience level.

The key factors influencing the resilience of the forest ecosystem varied among different areas, these spatial patterns of which may provide significant information for formulating appropriate forest resource management measures. For example, in the southwestern mountain area, the stand condition was not favorable due primarily to the terrain, which makes this area susceptible to soil erosion. However, the rich ecological memory found in this area substantially offsets this disadvantage. The patch area, with a weight of 0.1744 , is one of the most important indicators at the bottom level, and the patch area of most forest types was generally very large and the fragmentation was not serious in this area. By comparison, the stand condition is very favorable in the southeastern mountain area, with higher temperature and more precipitation that had favorable effects on the forests. However, the internal memory in this area only reached the medium level; only very few parts were rich in external memory. In the northern mountain area, especially in its western portion, the stand condition is not very good, and the ecological memory was only rich in a C. lanceolata forest extending from east to west due primarily to the abundant internal memory. However, the ecological memory of other forest types was very poor in this area, primarily due to the serious fragmentation. In the central plain and hill area, where there are primarily plain and low hills, the stand condition is very favorable for humans as well as forests. But this area has a long history of human disturbance, and the forests were seriously fragmented, leading to the very poor ecological memory.

The current spatial heterogeneity of the forest ecosystem resilience is the result of the cumulative effects of long-term 
human intervention and the influence of natural conditions [45]. The human disturbance plays a subordinate role in influencing the forest ecosystem resilience on the whole; however, it still may be the dominant influencing factor at the local scale. For example, there is still very serious human disturbance in the plain area, and the human activities still play a dominant role in influencing the forest ecosystem resilience, especially in the plain area in the middle part of the study area, where there is a high population density and a lot of forests have been reclaimed for cropland. In fact, the accumulative effects of historical human activities make great contribution to the current spatial pattern of the species composition of the forests. For example, the zonal vegetation is the evergreen broad-leaf forest, which has been seriously damaged during the historical period, and the gradual recovery of the local forests is due primarily to the reconstruction and conservation since the 1980s. As a result, C. lanceolata, which has been widely used in the afforestation projects, has accounted for the largest proportion of the forests at present. Overall, the result objectively reflects the spatial pattern of the forest ecosystem resilience in the study area, indicating that it is a practical approach to spatially measure the ecosystem resilience with its influencing factors.

3.2. Discussion. This study indicates that the ecosystem resilience can be quantitatively measured with its influencing factors; however, more efforts should be made to further explore how to more scientifically and accurately measure the ecosystem resilience since there are various challenges in both the spatially explicit assessment of the ecosystem resilience and its application. First and foremost, more efforts should be made to explore the theory and assessment of the ecosystem resilience. Although previous researches have provided some methods to measure the ecosystem resilience, there remains an urgent need for an operational tool for assessing and mapping the ecosystem resilience [46], and it is necessary to make more efforts to select and integrate the resilience indicators according to the research object and data availability based on firm theoretical foundations. Besides, methods for spatially assessing many resilience factors have not yet been developed, and the comparative study is crucial since the ecosystem resilience cannot always be directly observed [46]. In addition, since there may be multiple indicators to measure the ecosystem resilience, it is of great importance to explore how to integrate these indicators. The resilience was represented with weighted sum of indices of its influencing factors in this study; the result may be not very accurate since there may be some nonlinear relationship between the ecosystem resilience and its influencing factors, but it still provides a useful method to the spatially explicitly assess the ecosystem resilience.

There is also a great challenge to operationalize the resilience concept for ecosystem management in a dynamic world; one of the major challenges for progressing resiliencebased management lies in successful application. In fact, the general resilience principles have been influencing the way of the ecosystem management and conservation and have been consciously or unconsciously applied in the ecosystem management. For example, the biosphere reserves have been generally demarcated into core area(s), buffer area(s), and transition area(s) according to guidelines of the United Nations Educational, Scientific and Cultural Organization [47]. The demarcation of the biosphere reserves into three areas can promote the ecosystem resilience, although the ecosystem managers may have been not aware of that. For example, in fact there is a forest park in Sanwan Town where the forest ecosystem resilience is the highest, that is, Sanwan National Forest Park, which has a forest coverage rate of 90.5\% (Figure 2). This forest park was initially established in order to promote the development of the local tourism and pursue more economic benefit rather than improve the ecosystem resilience; however, it has unexpectedly increased the resilience of local forests. Therefore, establishing forest parks may be an effective way to conserve ecosystems and maintain the desirable ecosystem state.

\section{Conclusions}

Drawing on principles of the ecosystem resilience highlighted in the literature this study selected the indicators that capture the influencing factors of the ecosystem resilience and quantitatively assessed the forest ecosystem resilience in Yongxin County. The result indicates that it is feasible to generate large-scale ecosystem resilience maps with this assessment model and spatially explicitly identify the areas essential to the ecosystem conservation. Besides, the result show that there is significant spatial heterogeneity of the forest ecosystem resilience in the study area, which can provide a scientific basis for the local forest resource management and conservation to maintain critical ecosystem services and adapt to the climate change. But it is still necessary to make further improvement in the future research since there are still some controversies on the selection and integration of the specific indicators of ecosystem resilience, and it is possible to more accurately measure the ecosystem resilience if high resolution data in a large area are available. Although there are still some uncertainties, the results still can provide a scientific basis for the conservation of the forests, which is of great significance to the climate change mitigation.

\section{Conflict of Interests}

The authors declare that there is no conflict of interests regarding the publication of this paper.

\section{Acknowledgments}

This research was supported by the Project of the National Natural Science Foundation of China (Grant no. 41071343), the National Basic Research Program of China (973 Program) (Grant no. 2010CB950900), and the Key Project in the National Science \& Technology Pillar Program of China (no. 2013BAC03B00). Data support from the projects funded by the National Natural Science Foundation of China (Grant no. 71225005 , and Grant no. 40801231) is also greatly appreciated. 


\section{References}

[1] T. Prato, "Increasing resilience of natural protected areas to future climate change: a fuzzy adaptive management approach," Ecological Modelling, vol. 242, no. 1, pp. 46-53, 2012.

[2] E. J. Ma, A. P. Liu, X. Li, F. Wu, and J. Y. Zhan, "Impacts of vegetation change on the regional surface climate: a scenariobased analysis of afforestation in Jiangxi province, China," Advances in Meteorology, vol. 2013, Article ID 796163, 8 pages, 2013.

[3] I. Thompson, B. Mackey, S. Mcnulty, and A. Mosseler, Forest Resilience, Biodiversity, and Climate Change. A Synthesis of the Biodiversity/Resilience/Stability Relationship in Forest Ecosystems, Secretariat of the Convention on Biological Diversity, Montreal, Canada, 2009.

[4] Intergovernmental Panel on Climate Change (IPCC), Climate Change 2007: The Physical Science Basis, Cambridge University Press, Cambridge, UK, 2007.

[5] F. Brand, "Critical natural capital revisited: ecological resilience and sustainable development," Ecological Economics, vol. 68, no. 3, pp. 605-612, 2009.

[6] F. S. Chapin III, K. Danell, T. Elmqvist, C. Folke, and N. Fresco, "Managing climate change impacts to enhance the resilience and sustainability of Fennoscandian forests," AMBIO, vol. 36, no. 7, pp. 528-533, 2007.

[7] C. Folke, S. Carpenter, T. Elmqvist, L. Gunderson, C. S. Holling, and B. Walker, "Resilience and sustainable development: building adaptive capacity in a world of transformations," AMBIO, vol. 31, no. 5, pp. 437-440, 2002.

[8] J. K. Strickland-Munro, H. E. Allison, and S. A. Moore, "Using resilience concepts to investigate the impacts of protected area tourism on communities," Annals of Tourism Research, vol. 37, no. 2, pp. 499-519, 2010.

[9] G. C. Gallopín, "Linkages between vulnerability, resilience, and adaptive capacity," Global Environmental Change, vol. 16, no. 3, pp. 293-303, 2006.

[10] C. Hawes and C. Reed, "Theoretical steps towards modelling resilience in complex systems," in Computational Science and Its Applications-ICCSA 2006, Lecture Notes in Computer Science, pp. 644-653, Springer, Berlin, Germany, 2006.

[11] R. Macarthur, "Fluctuations of animal populations and a measure of community stability," Ecology, vol. 36, no. 3, pp. 533-536, 1955.

[12] D. R. Bellwood, A. S. Hoey, and J. H. Choat, "Limited functional redundancy in high diversity systems: resilience and ecosystem function on coral reefs," Ecology Letters, vol. 6, no. 4, pp. 281285, 2003.

[13] B. H. Walker, "Biodiversity and ecological redundancy," Conservation Biology, vol. 6, no. 1, pp. 18-23, 1992.

[14] J. C. Moore, P. C. de Ruiter, and H. W. Hunt, "Influence of productivity on the stability of real and model ecosystems," Science, vol. 261, no. 5123, pp. 906-908, 1993.

[15] V. L. Scarborough and W. R. Burnside, "Complexity and sustainability: perspectives from the ancient Maya and the modern Balinese," American Antiquity, vol. 75, no. 2, pp. 327363, 2010.

[16] G. Nicolis, I. Prigogine, and P. Carruthers, Exploring Complexity: An Introduction, W. H. Freeman, New York, NY, USA, 1989.

[17] S. Carpenter, Alternate States of Ecosystems: Evidence and Some Implications, Cambridge University Press, New York, NY, USA, 2001.
[18] R. Costanza, "Ecosystem health and ecological engineering," Ecological Engineering, vol. 45, pp. 24-29, 2012.

[19] E. M. Bennett, G. S. Cumming, and G. D. Peterson, "A systems model approach to determining resilience surrogates for case studies," Ecosystems, vol. 8, no. 8, pp. 945-957, 2005.

[20] O. J. Schmitz, "Combining field experiments and individualbased modeling to identify the dynamically relevant organizational scale in a field system," Oikos, vol. 89, no. 3, pp. 471-484, 2000.

[21] J. F. Franklin and J. A. MacMahon, "Message from a mountain," Science, vol. 288, no. 5469, pp. 1183-1185, 2000.

[22] M. T. Gibbs, "Resilience: what is it and what does it mean for marine policymakers?” Marine Policy, vol. 33, no. 2, pp. 322331, 2009.

[23] V. Rosset and B. Oertli, "Freshwater biodiversity under climate warming pressure: identifying the winners and losers in temperate standing waterbodies," Biological Conservation, vol. 144, no. 9, pp. 2311-2319, 2011.

[24] X. Z. Deng, J. K. Huang, E. Uchida, S. Rozelle, and J. Gibson, "Pressure cookers or pressure valves: do roads lead to deforestation in China?" Journal of Environmental Economics and Management, vol. 61, no. 1, pp. 79-94, 2011.

[25] X. Z. Deng, F. Yin, E. Uchida, and S. Rozelle, "A complementary measurement of changes in China's forestry area using remote sensing data," Journal of Food, Agriculture \& Environment, vol. 10, no. 3-4, pp. 1355-1358, 2012.

[26] F. Wu, J. Y. Zhan, H. M. Yan, C. C. Shi, and J. Huang, "Land cover mapping based on multisource spatial data mining approach for climate simulation: a case study in the farming-pastoral ecotone of North China," Advances in Meteorology, vol. 2013, Article ID 520803, 12 pages, 2013.

[27] Y. Jing, X. H. Yang, and D. Jiang, "The grid scale effect analysis on town leveled population statistical data spatialization," Journal of Geo-Information Science, vol. 12, no. 1, pp. 40-47, 2010.

[28] B. Wolfslehner, H. Vacik, and M. J. Lexer, "Application of the analytic network process in multi-criteria analysis of sustainable forest management," Forest Ecology and Management, vol. 207, no. 1-2, pp. 157-170, 2005.

[29] C. Folke, "Resilience: the emergence of a perspective for socialecological systems analyses," Global Environmental Change, vol. 16, no. 3, pp. 253-267, 2006.

[30] B. Walker, C. S. Holling, S. R. Carpenter, and A. Kinzig, "Resilience, adaptability and transformability in socialecological systems," Ecology and Society, vol. 9, no. 2, article 5, 2004.

[31] A. Jentsch, C. Beierkuhnlein, and P. S. White, "Scale, the dynamic stability of forest ecosystems, and the persistence of biodiversity," Silva Fennica, vol. 36, no. 1, pp. 393-400, 2002.

[32] D. Zirlewagen, G. Raben, and M. Weise, "Zoning of forest health conditions based on a set of soil, topographic and vegetation parameters," Forest Ecology and Management, vol. 248, no. 1-2, pp. 43-55, 2007.

[33] T. J. Martin and J. Ogden, "The seed ecology of Ascarina lucida: a rare New Zealand tree adapted to disturbance," New Zealand Journal of Botany, vol. 40, no. 3, pp. 397-404, 2002.

[34] S. Carpenter, B. Walker, J. M. Anderies, and N. Abel, "From metaphor to measurement: resilience of what to what?" Ecosystems, vol. 4, no. 8, pp. 765-781, 2001.

[35] Q. L. Shi, J. Y. Zhan, F. Wu, X. Z. Deng, and L. R. Xu, "Simulation on water flow and water quality in Wuliangsuhai lake using a 2-D hydrodynamic model," Journal of Food, Agriculture \& Environment, vol. 10, no. 2, pp. 973-975, 2012. 
[36] H. T. Dublin, A. R. E. Sinclair, and J. McGlade, "Elephants and fire as causes of multiple stable states in the Serengeti-Mara woodlands," Journal of Animal Ecology, vol. 59, no. 3, pp. 11471164, 1990.

[37] J. Bengtsson, P. Angelstam, T. Elmqvist et al., "Reserves, resilience and dynamic landscapes," $A M B I O$, vol. 32, no. 6, pp. 389-396, 2003.

[38] M. Nyström and C. Folke, "Spatial resilience of coral reefs," Ecosystems, vol. 4, no. 5, pp. 406-417, 2001.

[39] A. B. Khattak, A. Zeb, and N. Bibi, "Impact of germination time and type of illumination on carotenoidcontent, protein solubility and in vitro protein digestibility of chickpea(Cicer arietinum L.) sprouts," Food Chemistry, vol. 109, no. 4, pp. 797801,2008

[40] F. Berkes and D. Jolly, "Adapting to climate change: socialecological resilience in a Canadian western Arctic community," Conservation Ecology, vol. 5, no. 2, 2002.

[41] J. Ehrlén and O. Eriksson, "Dispersal limitation and patch occupancy in forest herbs," Ecology, vol. 81, no. 6, pp. 1667-1674, 2000.

[42] A. Borja, S. B. Bricker, D. M. Dauer et al., "Overview of integrative tools and methods in assessing ecological integrity in estuarine and coastal systems worldwide," Marine Pollution Bulletin, vol. 56, no. 9, pp. 1519-1537, 2008.

[43] N. Mantua, "Methods for detecting regime shifts in large marine ecosystems: a review with approaches applied to North Pacific data," Progress in Oceanography, vol. 60, no. 2-4, pp. 165-182, 2004.

[44] R. C. Rooney and S. E. Bayley, "Quantifying a stress gradient: an objective approach to variable selection, standardization and weighting in ecosystem assessment," Ecological Indicators, vol. 10, no. 6, pp. 1174-1183, 2010.

[45] J. Y. Zhan, N. N. Shi, S. J. He, and Y. Z. Lin, "Factors and mechanism driving the land-use conversion in Jiangxi province," Journal of Geographical Sciences, vol. 20, no. 4, pp. 525-539, 2010.

[46] G. Rowlands, S. Purkis, B. Riegl, L. Metsamaa, A. Bruckner, and P. Renaud, "Satellite imaging coral reef resilience at regional scale. A case-study from Saudi Arabia," Marine Pollution Bulletin, vol. 64, no. 6, pp. 1222-1237, 2012.

[47] UNESCO, "Action plan for biosphere reserves," Nature and Resources, vol. 12, no. 1, pp. 1-12, 1984. 

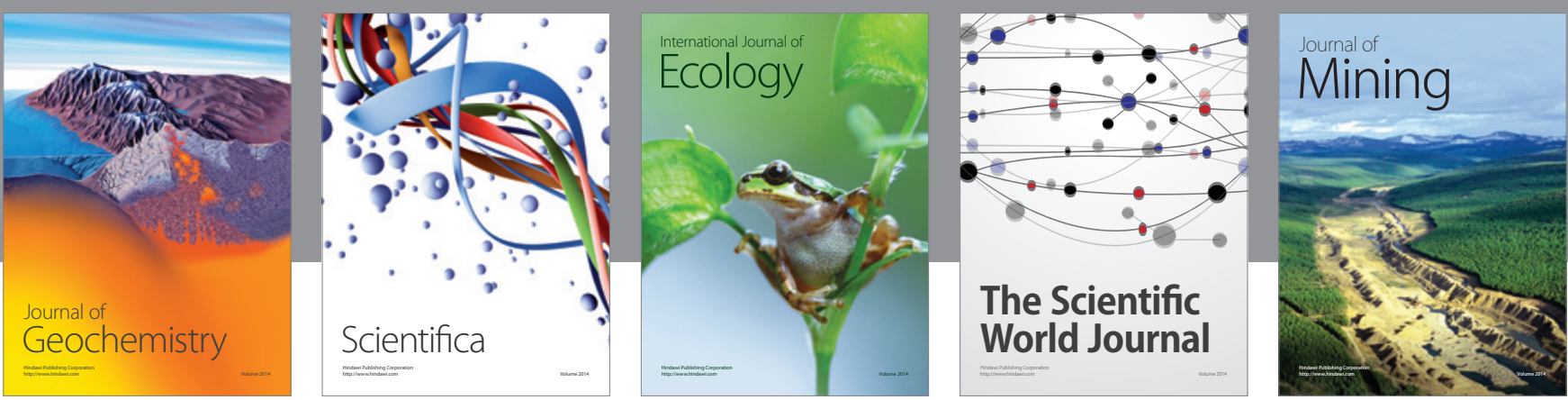

The Scientific World Journal
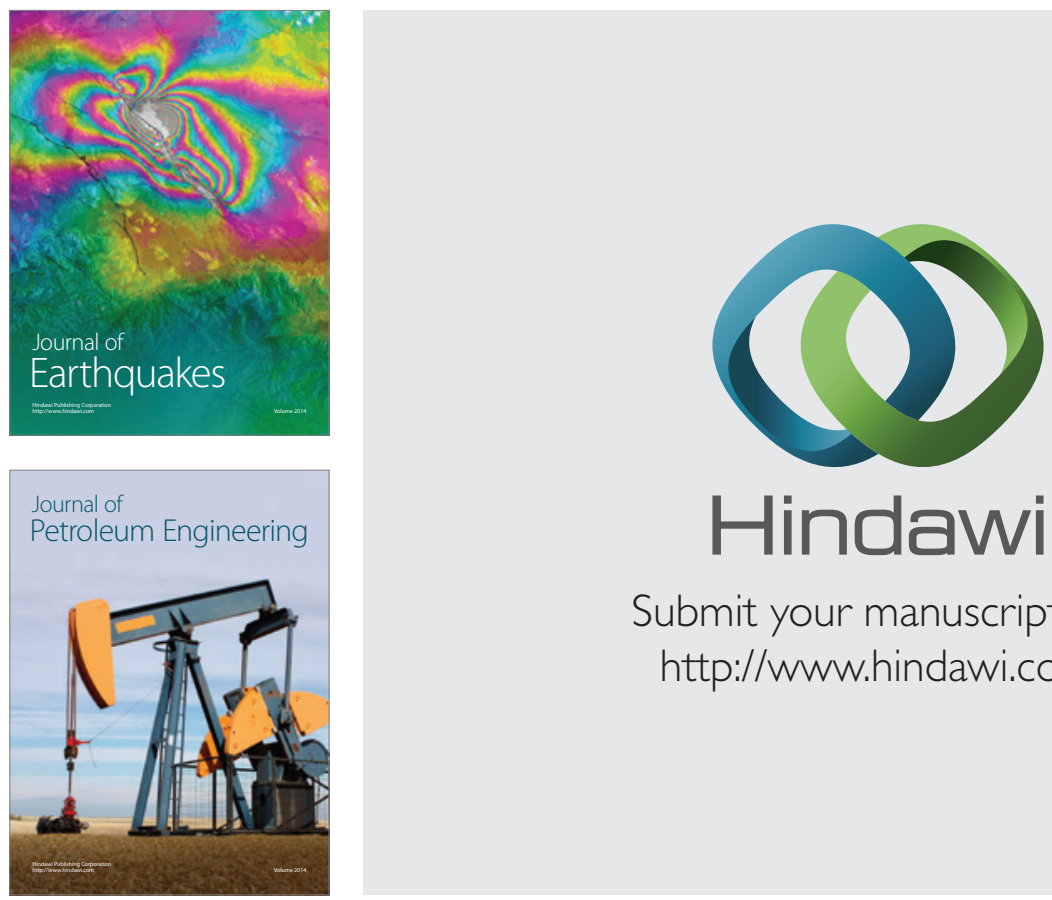

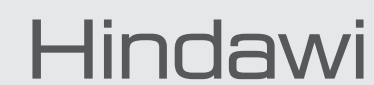

Submit your manuscripts at

http://www.hindawi.com
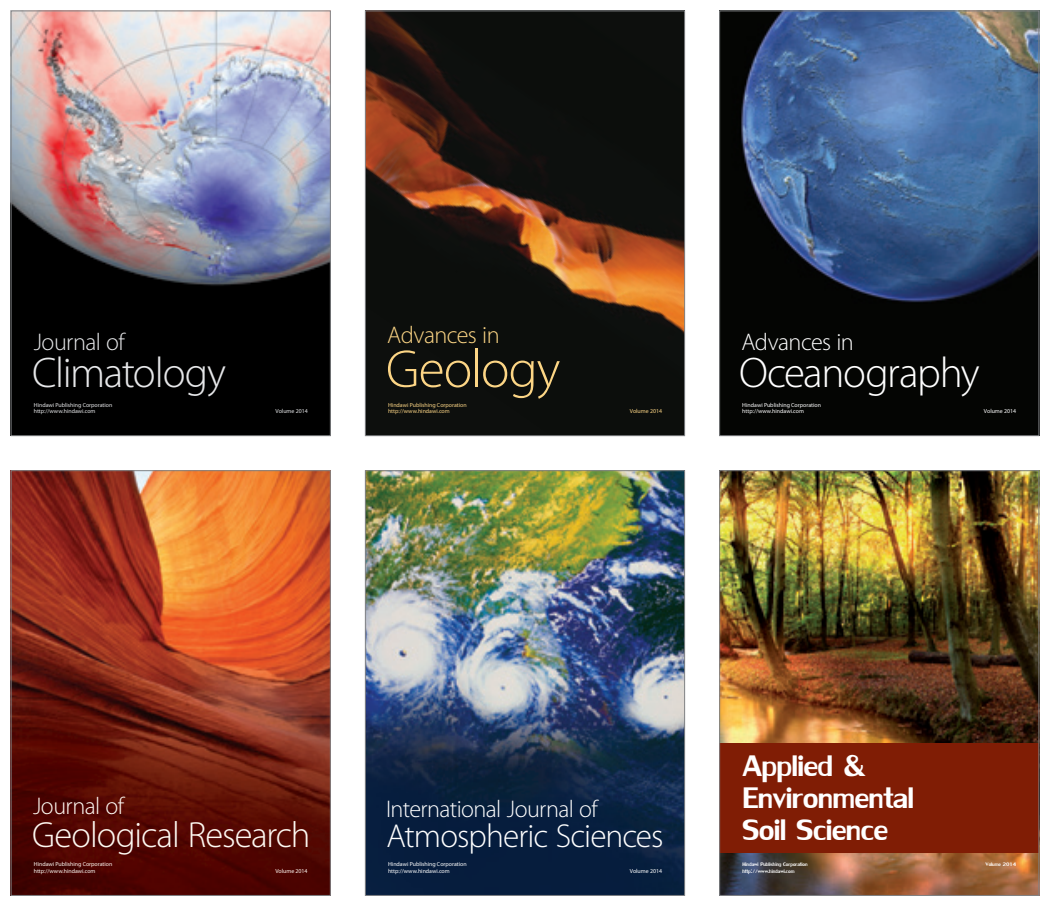
